\title{
With enzalutamide for prostate cancer, it may all depend on the tumor's AR profile
}

\author{
John A. Fracchia, MD \\ Lenox Hill Hospital, New York
}

See Community Translations on page 135

$\mathrm{E}$ nthusiasm continues in the medical community anxious for effective agents for men with metastatic castration-resistant prostate cancer (mCRPC). Most prostate cancer patients who develop metastatic disease are initially treated with readily available luteinizing hormone-releasing hormone (LHRH) agonists or antagonists, with or without an anti-androgen. The rationale here is to decrease androgen levels and/or block androgen receptor (AR) binding. In patients whose disease becomes refractory to this front-line hormonal deprivation, the molecular mechanisms involved in androgen independence include androgen receptor gene amplification, AR mutations that allow stimulation by a variety of weak androgens, and $\mathrm{AR}$ activation by autocrine production of androgens from tumor cells. Patients with metastatic castration-resistant prostate cancer (mCRPC) who biochemically recur after androgendeprivation therapy with significant prostate-specific antigen (PSA) elevations and/or who develop radiographic or symptomatic metastases are then usually considered for cytotoxic chemotherapy.

The only approved initial cytotoxic chemotherapeutic agent that has demonstrated improved survival for patients with $\mathrm{mCRPC}$ is docetaxel. For patients who fail docetaxel, cabazitaxel with prednisone is an approved second-line treatment. Similarly, another approved second-line treatment (albeit, hormonal) for patients who have failed docetaxel therapy is abiraterone acetate, which attacks the adrenal and extragonadal synthesis of androgen. The magnitude of this effect was not appreciated until it was recognized that the androgen receptor and ligand-dependent androgen receptor signaling remain active and upregulated in men with castrate levels of testosterone ( $\leq 50$ $\mathrm{ng} / \mathrm{dL}){ }^{1}$ Recognition of the importance of the signaling of the androgen receptor and its seemingly independent behavior in a milieu of little or no androgen is now appreciated. Chen et al. reported in 2004 on the

Correspondence John A. Fracchia, MD, 245 East 54th Street, New York, NY 10022 (drfracchia@nyurological.com).

Disclosures Dr. Fracchia has no disclosures. development of a second-generation potent oral nonsteroidal antiandrogen (MDV3110, ie, enzalutamide). ${ }^{2}$ This agent blocks AR binding with testosterone, blocks nuclear translocation of the AR from the cytoplasm, and blocks DNA binding and activation. ${ }^{3}$ It is novel in that unlike the established antiandrogens (bicalutamide, flutamide, nilutamide), it binds potently to the $\mathrm{AR}$, does not stimulate the AR in $\mathrm{mCRPC}$, has no measurable agonistic activity, and induces death in prostate cancer cells that were previously resistant to hormone-based therapies.

Scher et al have reported on 1,199 patients in the AFFIRM trial in which the primary endpoint was to evaluate survival in patients who had been previously treated with docetaxel and were randomized to receive placebo or enzalutamide. ${ }^{4}$ In addition to showing significant increases in survival - 18.4 months in the enzalutamide group, compared with 13.6 months in the placebo group - the study also demonstrated improved secondary endpoints (PSA response rates, soft-tissue response rates, time to progression, radiographic progressionfree survival). In addition, the incidence of high-grade side effects was higher in the placebo group, which suggested that enzalutamide was relatively more effective in ameliorating disease-related symptoms than was placebo. However, the agent does cross the blood-brain barrier and 5 patients $(0.6 \%)$ in the enzalutamdide group experienced seizures, which generated concern for use of the drug in patients with nonmetastatic central nervous system issues.

Another exciting trial of enzalutamide, which was fully enrolled in 2012 in advance of its anticipated end enroll date (2013), is the PREVAIL trial. ${ }^{5}$ This is a phase 3 multinational, double-blind, placebo-controlled study of patients at 222 study sites. The purpose of this randomized trial is to learn of potential benefit in overall survival and progression-free survival in chemotherapy-naïve men with progressive metastatic prostate cancer who have failed front-line androgen- (c) 2013 Frontline Medical Communications 


\section{Commentary}

deprivation therapy and who were randomized to receive either enzalutamide or placebo. The results of this trial and subsequent analysis should be available later this year or in early 2014. In addition, with our knowledge now of the importance of the AR receptor and any available androgen, there is impetus for a trial of a novel AR target signaling inhibitor such as enzalutamide with an androgen synthesis inhibitor such as abiraterone.

As promising as this novel AR inhibitor enzalutamide seems to be, we may have to temper some of our enthusiasm as it is unlikely that all men with mCRPC will benefit. About $25 \%$ of the tumors that were analyzed from men with $\mathrm{mCRPC}$ had no androgen receptors and demonstrated marked upregulation of mitotic genes independent of AR regulation. ${ }^{6}$ Nonetheless, it appears that we are making progress in extending over- all and progression-free survival through multiple approaches on an array of targets.

\section{References}

1. Debes JD, Tindall DJ. Mechanisms of androgen-refractory prostate cancer. N Engl J Med. 2004;351:1488-1490.

2. Chen Y, Clegg NJ, Scher HI. Anti-androgens and androgendepleting therapies in prostate cancer: new agents for an established target. Lancet Oncol. 2009;10:981-991.

3. Tran C, Ouk S, Clegg NJ, et al. Development of a second-generation antiandrogen for treatment of advanced prostate cancer. Science. 2009;324:787-790.

4. Scher HI, Fizazi K, Saad F, et al.; AFFIRM Investigators. $N$ Engl J Med. 2012;367(13):1187-1189.

5. National Institutes of Health. A safety and efficacy study of oral MDV3100 in chemotherapy-naive patients with progressive metastatic prostate cancer (PREVAIL). http://clinicaltrials.gov/ show/NCT01212991. Updated January 29, 2023. Accessed April 15,2013

6. Tzelepi V, Zhang J, LU JF, et al. Modeling a lethal prostate cancer variant with small-cell carcinoma features. Clin Cancer Res. 2012; 18:666-677. 